\title{
Bayesian Estimation of Intrinsic Tissue Oxygenation and Perfusion from RGB Images
}

\author{
Geoffrey Jones $^{1}$, Neil T Clancy ${ }^{2,3}$, Yusuf Helo ${ }^{1}$, Simon Arridge ${ }^{2}$, Daniel S Elson ${ }^{2,3}$, Danail Stoyanov ${ }^{1}$
}

\begin{abstract}
Multispectral imaging (MSI) can potentially assist the intra-operative assessment of tissue structure, function and viability, by providing information about oxygenation. In this paper, we present a novel technique for recovering intrinsic MSI measurements from endoscopic RGB images without custom hardware adaptations. The advantage of this approach is that it requires no modification to existing surgical and diagnostic endoscopic imaging systems. Our method uses a radiometric colour calibration of the endoscopic camera's sensor in conjunction with a Bayesian framework to recover a per-pixel measurement of the total blood volume (THb) and oxygen saturation $\left(\mathrm{SO}_{2}\right)$ in the observed tissue. The sensor's pixel measurements are modelled as weighted sums over a mixture of Poisson distributions and we optimise the variables $\mathrm{SO}_{2}$ and $\mathrm{THb}$ to maximise the likelihood of the observations. To validate our technique, we use synthetic images generated from Monte Carlo (MC) physics simulation of light transport through soft tissue containing sub-surface blood vessels. We also validate our method on in vivo data by comparing it to a MSI dataset acquired with a hardware system that sequentially images multiple spectral bands without overlap. Our results are promising and show that we are able to provide surgeons with additional relevant information by processing endoscopic images with our modelling and inference framework.
\end{abstract}

Index Terms-Multispectral Imaging, Minimally Invasive Surgery, Bayesian Inference, Biophotonics, Surgical Vision

\section{INTRODUCTION}

I NTRA-operative imaging during minimally invasive surgery (MIS) is the primary method of sensing used to guide surgical actions, and MIS is feasible today largely due to the evolution of digital imaging technologies that make it possible to deliver and harvest light from the surgical site through an endoscope [1]. During MIS, access to the surgical site is restricted by the size of the entry ports [2], this makes it challenging to interrogate the structure and function of tissues during surgery. Currently, surgeons are primarily reliant on images acquired in the visible light spectrum using three channel (RGB) sensors [3] either mounted at the tip of the endoscope or attached to the distal end and linked to lenses or fibre bundles. Such endoscopic system designs provide realtime images of the tissue surface that are used to guide diagnostic and therapeutic procedures. The drawback of imaging

Manuscript received December 23 2016; revised February 5, 2017. ${ }^{1}$ Centre for Medical Image Computing, University College London (web: http://cmic. cs.ucl.ac.uk),

${ }^{2}$ Department of Surgery and Cancer, Imperial College London

3 The Hamlyn Centre, Imperial College London (web: http://imperial.ac. uk/hamlyn )

Copyright (C) 2010 IEEE. Personal use of this material is permitted. However, permission to use this material for any other purposes must be obtained from the IEEE by sending a request to pubs-permissions@ieee.org. in this way is that it provides limited information about the functional characteristic of the tissue. This makes it difficult for surgeons to assess tissue viability, for example to evaluate posttransplantation revascularisation, or to characterise tissue types for diagnostic decisions.

To capture this information complementary imaging modalities can be introduced. The endoscope itself can be augmented, for example by altering the sensitivity of the imaging sensor or by changing the method in which light is delivered to the surgical site [4] [5]. Imaging agents can also be used to highlight structures. However, the use of additional imaging hardware or the injection of agents, introduces complexity to the regulatory requirements of the system and adds to the cost of new devices. Therefore making the most effective use of the available information within current endoscopic imaging solutions is crucial and can have important clinical significance. Image processing on the endoscopic RGB data is intrinsically registered to the surgeon's view of the surgical site and video is typically captured at high frame rate enabling dynamic imaging in the presence of physiological tissue motion. The problem is however ill posed because the RGB data provided by the endoscope is a limited measurement of a complex process relating the interaction between light and tissue to clinically relevant parameters.

In this paper we use the observation that, within tissue, the dominant absorber of light in the visible wavelength range is haemoglobin [6]. This allows us to formulate a prior on the expected spectrum of light observed in endoscopic images. We use this prior to regularise the estimation of tissue perfusion and oxygenation from endoscopic RGB images. Our method works on a per pixel level but can be used as a framework that underpins the incorporation of both temporal and spatial regularisation terms.

\section{RELATED WORK IN MSI FOR SURGERY}

Pathological signals such as oxy- and deoxy-haemoglobin ( $\mathrm{HbO}$ and $\mathrm{Hb}$ ) concentration, often correspond to tissue structure and viability [8]. These chromophores can be detected from their characteristic attenuation of light in the visible wavelength range [9]. As shown in Fig. 1b the attenuation spectra for $\mathrm{HbO}$ and $\mathrm{Hb}$ are quite close in places $(500 \mathrm{~nm})$ while varying a lot in others $(650 \mathrm{~nm})$ enabling inference to be made of their relative and combined concentrations respectively. This response can be imaged by using sequential MSI where multiple narrow band images are acquired. MSI hardware has been used for multiple clinical indications, such as the assessment of ischaemia in heart tissue without ionising 


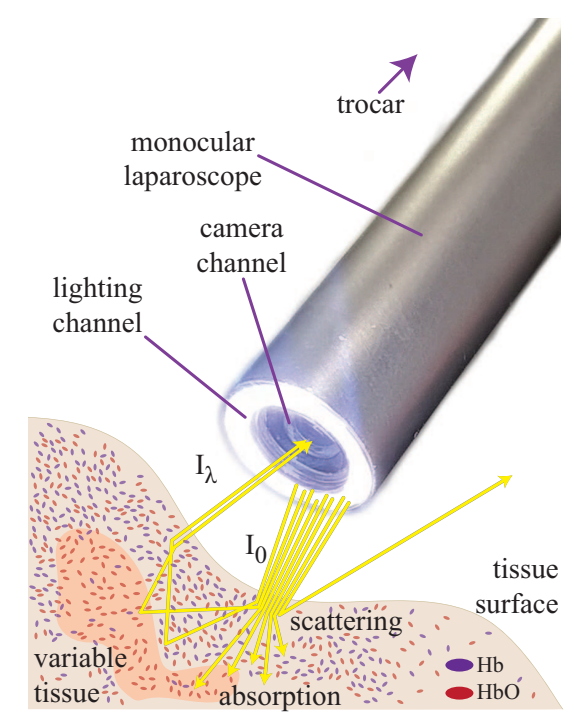

(a)

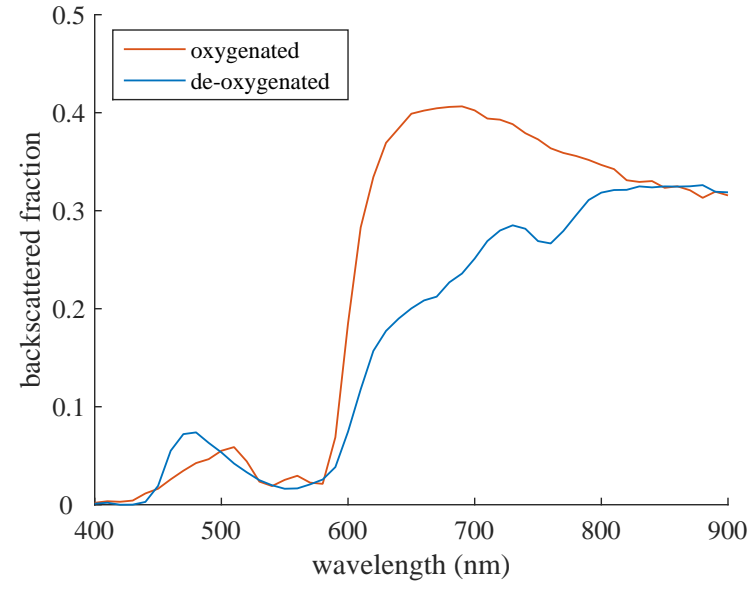

(b)

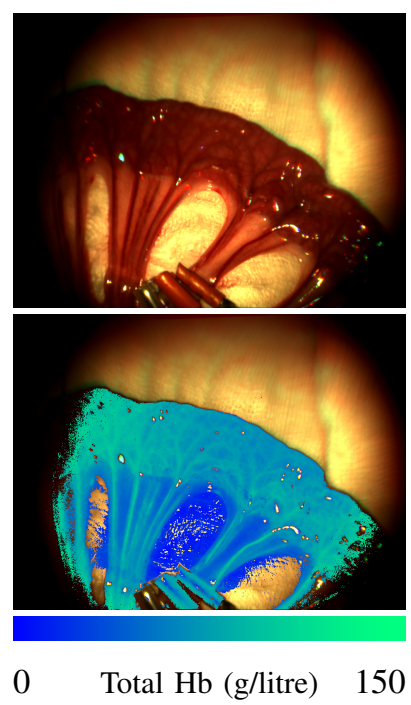

(c)

Fig. 1: (a) The interaction between light and tissue comprises both scattering and absorption and the inhomogeneity in the concentration of chromophores results in a spatially varying distribution of detected light. The effective attenuation of the measured light in an endoscopic image is a combination of these effects. (b) The backscattered fraction of light for oxygenated and de-oxygenated blood under uniform illumination generated by $\mathrm{MC}$ simulation [7]. This provides an aggregate attenuation coefficient $(-\log (\cdot))$ that combines the attenuation due to absorption and scattering as well as the mean travel distance within tissue. (c) The total haemoglobin ( $\mathrm{THb}$ ) is a measure formed by the sum of concentrations of these two chromophores and saturation is the ratio of oxygenated blood to total haemoglobin. These can be superimposed on endoscopic images to provide the surgeon with functional information during an intervention.

radiation [10] or in eye surgery to minimise photo toxicity [11]. Endoscopic MSI has also been used to identify abnormalities by detecting the increased vascularisation of malignant tissue [12] and to assess the success of organ transplantation by inference of $\mathrm{SO}_{2}$ and $\mathrm{THb}$ posttransplantation [9].

Hardware approaches to MSI can involve rapidly varying illumination [13], liquid crystal tunable filters (LCTF) [14] or fast filter wheels [15]. The degree of illuminant attenuation by tissue is measured in multiple non-overlapping spectral bands, and the Beer-Lambert equation is used to relate these attenuation values to chromophore concentrations. Given assumptions that individual band measurements are each across a narrow spectral range, without overlapping each other and that there is no fluorescence and negligible constant scattering, estimating chromophore concentrations becomes a linear fitting problem. While having a large number of spectral bands enables good fitting results, it often comes at the cost of the reduced temporal resolution of acquiring the whole multispectral cube, making it difficult to image tissue intra-operatively due to physiological motion [16], [17], [18], [19]. Filter wheel and strobe illumination can allow for rapid data acquisition, while LCTF approaches typically provide more fine spectral data.

To estimate chromophore concentrations from RGB endoscopic image data in the same way as for MSI data is not possible. This is because RGB data comprises three wide spectral bands, characterised by the spectral response curves of the RGB sensor, often having a high degree of overlap between bands. However, it has previously been shown for near infrared wavelengths that there is strong colour correspondence in CIE $1976 \mathrm{~L}^{*} \mathrm{a} * \mathrm{~b} *$ colour space for simulated [20] and observed tissue types for a range of tissue models [21], and $\mathrm{HbO}$ and $\mathrm{Hb}$ could be similarly detectable. Recently it has been demonstrated that RGB data can be used to estimate MSI data by Wiener filtering [22] from which $\mathrm{THb}(\mathrm{HbO}+\mathrm{Hb})$ and $\mathrm{SO}_{2}\left(\frac{\mathrm{HbO}}{\mathrm{THb}}\right)$ can be calculated using Beer-Lambert based fitting. The method shows very promising results, however, it relies on learning the characteristic reflectance spectra specific to a singular tissue type. Alternatively the overlap in the RGB data can be minimised and more precisely related by integrating a filter wheel, in front of the RGB camera, containing triple band pass filters. Using such a configuration it is possible to record a set of 18 narrow spectral band filtered measurements with six RGB images [23], these can then be used to estimate the MSI data knowing the filter transmission spectra and RGB camera response curves. This framework accounts for spatially varying geometry and achieves a frame rate of $15 \mathrm{fps}$ by a using a graphics processing unit (GPU) implementation of linear matrix inversion to estimate $\mathrm{THb}$ and $\mathrm{HbO}$. The assumption that the latent spectral power distribution of light arriving at the camera is smooth across wavelengths can also be used to develop a Tikhonov regularisation to constrain estimation MSI [24]. While being computationally efficient and promising for $\mathrm{THb}$ estimation, the smoothing comes at the cost of being able to accurately differentiate between $\mathrm{HbO}$ and $\mathrm{Hb}$. 


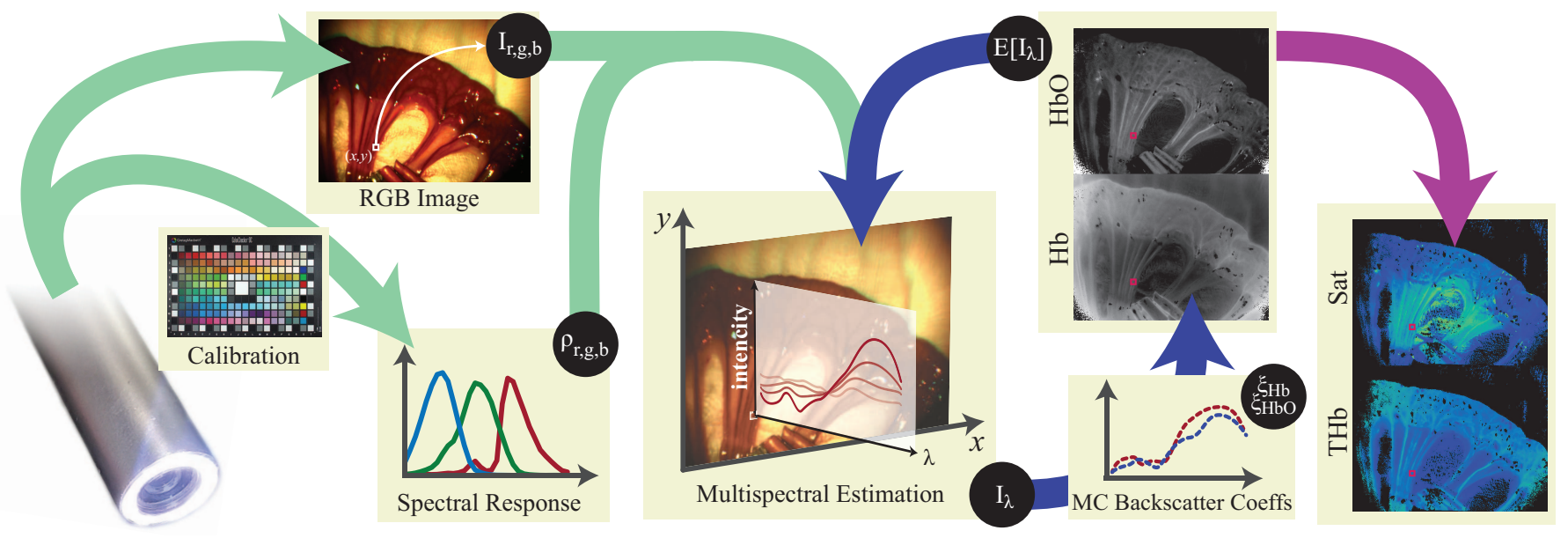

Fig. 2: We capture a spectral calibration for the endoscopic sensor by using a colour chart illuminated with standard lighting. When we capture an RGB image from the scope for each pixel we alternately estimate the multispectral signal from the RGB camera calibration and the current concentrations estimate (detail in Fig. 3). We then update the concentrations estimate from the current multispectral estimate and the MC computed backscatter data.

\section{METHOD}

\section{A. Light-Tissue Reflectance Model}

Light arriving at the endoscopic camera (Fig. 1) is assumed to have been back scattered and attenuated by passing through tissue containing spatially varying concentrations of chromophores. The dominant chromophores in tissue are assumed to be the two oxygenation states that haemoglobin can take, $\mathrm{HbO}$ and $\mathrm{Hb}(\boldsymbol{h} \boldsymbol{b})$, corresponding to oxygenated and deoxygenated respectively. At a given $x, y$ image location $(\boldsymbol{x})$ the concentration of chromophores $\boldsymbol{h} \boldsymbol{b}(\boldsymbol{x})$, within the corresponding tissue location, can be estimated using the modified Beer-Lambert relationship [25]. This defines the model for how the concentration of all chromophores present in a sample (the tissue corresponding to image position $\boldsymbol{x}$ ) attenuate the incident light $\boldsymbol{I}_{0, \boldsymbol{\lambda}}(\boldsymbol{x})$ for a set of illumination wavelengths $\lambda$. The resulting intensity of light leaving the scene at that position, $\boldsymbol{I}_{\boldsymbol{\lambda}}(\boldsymbol{x})$ can then be calculated as:

$$
\boldsymbol{I}_{\boldsymbol{\lambda}}(\boldsymbol{x})=\boldsymbol{I}_{0, \boldsymbol{\lambda}}(\boldsymbol{x}) e^{\left(-\boldsymbol{G}(\boldsymbol{x})-\boldsymbol{h} \boldsymbol{b}(\boldsymbol{x}) \boldsymbol{\mu}_{\boldsymbol{h b}, \lambda} \xi_{h \boldsymbol{b}}\right)}
$$

From the original Beer-Lambert equation, we can express the attenuation due to absorption $\boldsymbol{\xi}_{\boldsymbol{h b}, \boldsymbol{\lambda}}$ over the wavelength range $\boldsymbol{\lambda}$ for each chromophore. The modified form of the BeerLambert relationship introduces two additions. The first new variable is the mean path length of light travelling through the tissue $\boldsymbol{\mu}_{\boldsymbol{h} \boldsymbol{b}, \boldsymbol{\lambda}}$. This is assumed to be longer than the shortest distance between the source and detector because each photon will have been scattered along the way. The assumption is appropriate for endoscopic systems where the illumination and the camera are modelled at the tip of the scope and also since each photon will take a slightly different path the value of $\boldsymbol{\mu}$ represents the mean path length. The second extension is to include a geometric factor $\boldsymbol{G}(\boldsymbol{x})$ to describe the effect of loss due to scattering such that the resultant photon path never intersects the detector. We use a single term representation but this geometry factor can also be expanded as a constant and linear term with the latter being dependant on wavelength [26].
Rearranging the terms of (1) it is possible to formulate a linear relationship:

$$
\mathrm{A}=-\log \left(\frac{\boldsymbol{I}_{\lambda}(\boldsymbol{x})}{\boldsymbol{I}_{0, \lambda}(\boldsymbol{x})}\right)=\boldsymbol{G}(\boldsymbol{x})+\boldsymbol{h} \boldsymbol{b}(\boldsymbol{x}) \boldsymbol{\mu}_{\boldsymbol{h} \boldsymbol{b}, \lambda} \boldsymbol{\xi}_{\boldsymbol{h b}} .
$$

The negative log ratio of the incident to exit intensity is the total attenuation, A. When the wavelength range is narrower, for example $500 \mathrm{~nm}$ to $600 \mathrm{~nm}$, the constant offset plus linear scattering term can be used for $\boldsymbol{G}(\boldsymbol{x})$ [26]. However, when using an RGB sensor, over the wide spectral range of each measurement, the scattering parameters for $\mathrm{HbO}$ and $\mathrm{Hb}$ are not completely linear. This observation is apparent on inspection of the scattering and attenuation coefficients data [7], especially towards shorter wavelengths, and varies slightly between each chromophore. For our formulation, instead of analytically modelling the wavelength dependent absorption and scattering attenuation for each chromophore, we empirically derive an attenuation factor $\hat{\xi}_{\boldsymbol{h} b}$ by using MC simulation [13], [27]. If we know the incident illumination, we can find the chromophore concentration parameters $\boldsymbol{h} \boldsymbol{b}(\boldsymbol{x})$ on the right by making many spectrally distinct measurements of the tissue and solving the linear equation:

$$
\mathrm{A}=\hat{\boldsymbol{\xi}}_{\boldsymbol{h} \boldsymbol{b}} \boldsymbol{h} \boldsymbol{b}(\boldsymbol{x})
$$

Approaches where many MSI narrow bands can be obtained, will typically use a form of (2) or (3) either with or without the scattering component. But for our estimation we adopt the above light-tissue interaction relationship.

\section{B. Sensor Model}

We assume that the probability for a sensor to detect photons is dependant on two random variables. The first is the probability that a photon left the scene and arrived at the sensor $\boldsymbol{I}(\boldsymbol{x}, \boldsymbol{h} \boldsymbol{b}(\boldsymbol{x}))$ and second describes whether the sensor will detect it $\rho$ based on its spectral sensitivity as shown in see Fig. 2. 


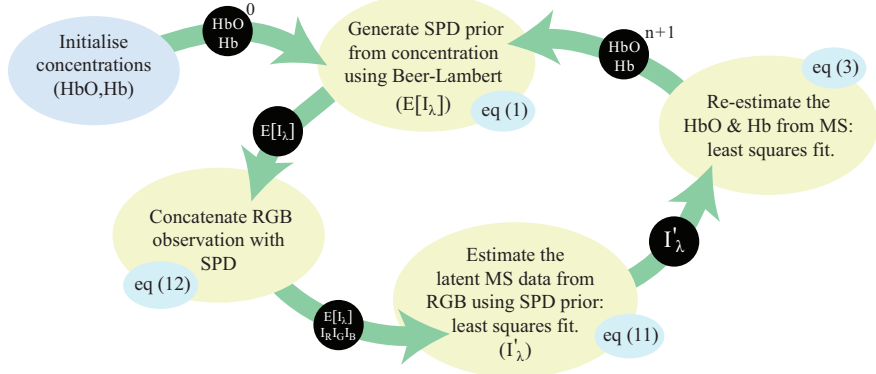

Fig. 3: Iterative Bayesian estimation of the concentrations alternately estimates the latent multispectral information $\boldsymbol{I}_{\lambda}^{\prime}$ regularised by the current concentration estimate, and subsequently updates the concentration estimate by fitting against $I_{\lambda}^{\prime}$.

We assume $\rho$ is constant throughout the imaging process but will vary dependent on wavelength. The resulting formulation becomes a counting problem and the probability distribution of the sensor becomes $\operatorname{Poisson}\left(\rho_{\lambda} \cdot \mathrm{E}\left[I_{\lambda}(\boldsymbol{x}, \boldsymbol{h} \boldsymbol{b}(\boldsymbol{x}))\right]\right)$ for any given wavelength, where $\mathrm{E}[\cdot]$ represents the expectation of the random variable.

For a wide band detector, such as an entire channel $(c)$ of an RGB sensor, the weighted sum over the sensitive range of wavelengths has weights corresponding to the sensitivity $\rho_{c, \lambda}$ at that particular wavelength. We can write the random variable of the channel measurement $\boldsymbol{I}_{c}(\boldsymbol{x}, \boldsymbol{h} \boldsymbol{b}(\boldsymbol{x}))$ as the inner product over all wavelengths:

$$
\boldsymbol{I}_{c}(\boldsymbol{x}, \boldsymbol{\xi}(\boldsymbol{x}))=\left\langle\boldsymbol{\rho}_{c}, \boldsymbol{I}(\boldsymbol{x}, \boldsymbol{h} \boldsymbol{b}(\boldsymbol{x}))\right\rangle_{\lambda}
$$

This is a straightforward sum because the contributions from different wavelengths are independent when conditioned on the chromophore concentration $\boldsymbol{h} \boldsymbol{b}(\boldsymbol{x})$. The distribution of this random variable is also Poisson distributed with a mean and variance:

$$
\mu_{c}=\sigma_{c}^{2}=\sum^{\lambda} \rho_{c, \lambda} \mathrm{E}\left[I_{\lambda}(\boldsymbol{x})\right]
$$

The random variables for sensor measurements are also conditional on the unknown concentration values $\boldsymbol{h} \boldsymbol{b}(\boldsymbol{x})$ at a given position.

\section{Reflectance Spectra Estimation}

Since (4) is a linear model, the natural way to recover $\boldsymbol{I}_{\lambda}(\boldsymbol{x}, \boldsymbol{h} \boldsymbol{b}(\boldsymbol{x}))$ is to use regularised linear inversion, regularisation being needed to account for (4) being ill conditioned. An attractive method to do so is Tikhonov regularisation, as it provides a closed form regularised estimation of the multispectral reflectance [24], [28]. Solving at point $\boldsymbol{x}$ (omitted for brevity $\left.{ }^{1}\right)$ as a linear system with a regularisation parameter $\gamma$ :

$$
\boldsymbol{I}_{\boldsymbol{\lambda}}=\left(\boldsymbol{\rho}^{\mathrm{T}} \boldsymbol{\rho}+\gamma * I d\right)^{-1} \boldsymbol{\rho}^{\mathrm{T}} \cdot \boldsymbol{I}_{\boldsymbol{c}}
$$

${ }^{1}$ From here on, we continue to omit the spatial variable $(\boldsymbol{x})$ that describes a position in the image because all calculations are local to a point without neighbourhood information.
Adopting normal notation $I d$ is the identity matrix. While using Tikhonov regularisation is computationally efficient, it often trades stability of the inversion at the cost of over smoothing the result, which introduces a bias in the computed haemoglobin concentrations. While it may be possible to determine a heuristic to correct for the resultant estimation bias, by fitting computed to known concentration, this would limit the flexibility and robustness of the method and would be unique for every sensor. To avoid having to calculate sensor specific correction factors, as well as to have greater resilience to sensor noise, we propose an alternative approach to estimation using Bayesian inference.

For an RGB sensor, Bayes' rule gives the relationship:

$$
P\left(\boldsymbol{h} \boldsymbol{b} \mid I_{r}, I_{g}, I_{b}\right)=\frac{P\left(I_{r}, I_{g}, I_{b} \mid \boldsymbol{h} \boldsymbol{b}\right) P(\boldsymbol{h} \boldsymbol{b})}{P\left(I_{r}, I_{g}, I_{b}\right)}
$$

where $P(\cdot)$ is a probability. Due to the channel response being independent and conditioned on the chromophore concentrations, we can formulate the right hand side of (7) as:

$$
\frac{P\left(I_{r} \mid \boldsymbol{h} \boldsymbol{b}\right) P\left(I_{g} \mid \boldsymbol{h} \boldsymbol{b}\right) P\left(I_{b} \mid \boldsymbol{h} \boldsymbol{b}\right) P(\boldsymbol{h} \boldsymbol{b})}{P\left(I_{r}, I_{g}, I_{b}\right)}
$$

This gives us the product of measurement probabilities for individual channels and a prior on the concentrations $\boldsymbol{h} \boldsymbol{b}$.

Since each channel accumulates over a wide wavelength range, the mean counts will be large and hence we can approximate the Poisson distribution of count values in each channel as normally distributed $\mathcal{N}(\mu, \sigma)$. Because the denominator of (8) does not depend on $\boldsymbol{h} \boldsymbol{b}$, we can ignore it for the purposes of maximisation, and we know then that (7) will be maximised for:

$$
\underset{\xi}{\arg \max }\left\{P(\boldsymbol{h} \boldsymbol{b}) \prod^{\boldsymbol{c}} \mathcal{N}\left(\boldsymbol{\mu}_{c, \boldsymbol{h} \boldsymbol{b}}, \boldsymbol{\sigma}_{c, \boldsymbol{h} \boldsymbol{b}}\right)\right\}
$$

The only prior on $\boldsymbol{h} \boldsymbol{b}$ are that all elements are non-negative because it is not possible to have negative concentrations. While this may seem too relaxed a constraint allowing concentrations to be potentially infinite, the cases when this might occur are limited to degenerate situations where the count for each channel of the sensor is zero or very close to zero.

\section{Parameter Optimisation}

Since the modified Beer-Lambert model is only suitable for measurements that are non-overlapping and narrow banded, we first estimate the spectrum of incidental light $\boldsymbol{I}_{\lambda}{ }^{\prime}$ arriving at the RGB sensor. Secondly we estimate the concentrations of $\mathrm{HbO}$ and $\mathrm{Hb}$ from $\boldsymbol{I}_{\lambda}^{\prime}$ solving (2) by minimising the sum of square difference (SSD). We perform this iteratively alternating between estimating the multispectral data $\boldsymbol{I}_{\lambda}{ }^{\prime}$ and then concentrations $\boldsymbol{h} \boldsymbol{b}$.

Estimating the multispectral data from RGB measurements by linearly solving using the camera response matrix results in a very poor approximation even with the positivity constraint. Typically this is because the formulation results in a sparse estimate with high values in a small number of wavelengths due to being highly under constrained. We can utilise a 
Bayesian maximum a posterior (MAP) estimation derived from (9), to pose this step in a constrained way as:

$$
\boldsymbol{I}_{\lambda}^{\prime}=\underset{\boldsymbol{I}_{\lambda}}{\arg \max }\left\{\left(\prod^{\boldsymbol{c}} \mathcal{N}\left(\boldsymbol{\mu}_{c, \boldsymbol{I}_{\lambda}}, \boldsymbol{\sigma}_{c, \boldsymbol{I}_{\lambda}}\right)\right) P\left(\boldsymbol{I}_{\boldsymbol{\lambda}}\right)\right\}
$$

The prior is generated from the previous estimation of $\boldsymbol{\xi}$ using (1) to estimate the absorption. Each per channel variable is maximised when the difference is minimised between $\left\langle\boldsymbol{\rho}_{c}, \boldsymbol{I}_{\lambda}\right\rangle$ and the measurements made in each channel. The effect of the prior is to penalise the per wavelength deviation of $\boldsymbol{I}_{\lambda}$ from the the current estimate hence we can pose as the minimisation:

$$
\boldsymbol{I}_{\lambda}^{\prime}=\underset{\boldsymbol{I}_{\lambda}}{\arg \min }\left\{\sum^{c}\left(\left\langle\boldsymbol{\rho}_{c}, \boldsymbol{I}_{\lambda}\right\rangle-I_{c}\right)^{2}+\sum^{\lambda}\left(\boldsymbol{I}_{\lambda}-\mathrm{E}\left[I_{\lambda}\right]\right)^{2}\right\}_{(11)}
$$

The expected value of the spectral power distribution (SPD) of $\boldsymbol{I}_{\lambda}$, written $\mathrm{E}\left[\boldsymbol{I}_{\lambda}\right]$, is derived generatively from (1) using the previous iterations' concentration estimates. It is important to note that the approximation of the Poisson distribution by the Normal distribution for large counts could potentially allow negative count values for each wavelength. To avoid this we impose a non-negativity constraint on $\boldsymbol{I}_{\lambda}^{\prime}$ to prevent physically impossible values. The full estimation and optimisation process can be summarised as shown in Fig. 2 .

\section{E. Implementation Details}

The optimisation of (11) is possible via a single linear expression solving for $\boldsymbol{I}_{\lambda}^{\prime}$ on the left hand side below:

$$
\left[\begin{array}{cccc}
\gamma & & 0 & \\
& \ddots & & \boldsymbol{\rho}_{r, g, b} \\
0 & & \gamma &
\end{array}\right] \boldsymbol{I}_{\lambda}^{\prime}=\left[\begin{array}{c}
\gamma \mathrm{E}\left[\boldsymbol{I}_{\lambda}\right] \\
I_{r} \\
I_{g} \\
I_{b}
\end{array}\right]
$$

where $I_{r}, I_{g}$ and $I_{b}$ are the sensor measurements in each channel and $\gamma$ is a regularisation constant. To prevent the prior dominating the term we use $\gamma=0.01$, this value of $\gamma$ was determined as effective experimentally via line search on synthetic data sets. As $\gamma$ affects the expression of the prior it is important that its value is not too small otherwise when solving (12) the left hand side will be ill conditioned. Similarly, too great a value will cause over fitting to the prior estimation ignoring the RGB measurements. Since the left hand side of (12) is constant over all iterations, a computationally efficient approach is to reuse decompositions of the left hand side matrix at each iteration. The two step estimation process of alternatively estimating the latent multispectral data and then the chromophone concentrations is illustrated in Fig. 3.

A fast non-negative least squares method is used to solve (12) and also for the subsequent parameter estimation from the estimated emission multispectral data [29]. Direct computation using our method with on-the-fly optimisation yields frame rates of 2.5 frames per second (720p), using a Surface Pro 3 2.3GHz 8GB RAM. However, the optimisation is deterministic for a given illumination spectrum and camera sensor response

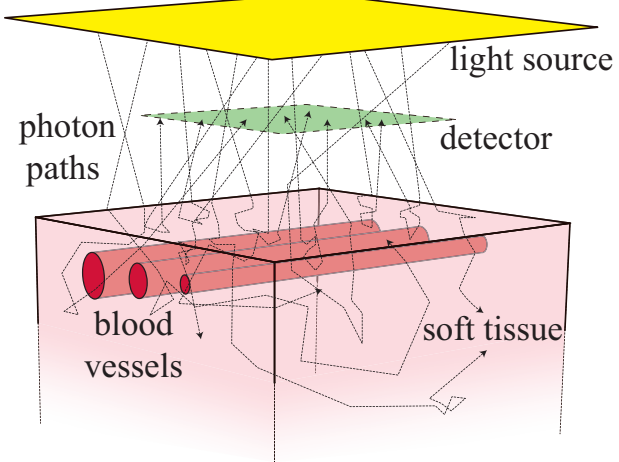

Fig. 4: Our experimental simulation model comprising an isotropic planar (40mm square) light source and a soft tissue volume $(100 \mathrm{~mm}$ cube). Inset in the tissue are three parallel blood vessels at constant depth. Photon trajectories are tracked until they leave the simulation volume or arrive at the detector (20mm wide square, parallel to, and $10 \mathrm{~mm}$ away from, the tissue surface).

and real-time performance can easily be achieved by using a precomputed look up table (LUT) of all possible RGB values. Using a LUT consisting of all possible 24bit RGB colours (approx. 16 million) we were able to process endoscopic images at video rate (over 30fps) using a serial implementation requiring $270 \mathrm{MB}$ of RAM.

\section{EXPERIMENTS AND RESULTS}

We report results for two types of validation, using simulated data and in vivo porcine data where we can see the practical validity of the approach. We generated simulated data to study the numerical stability and accuracy of our method in controlled scenarios. Our real experimental validation utilises data gathered in vivo from an animal experiment to evaluate the performance of our method compared to Tikhonov regularised estimation [24] and the result from using an endoscopic MSI device [14].

\section{A. Synthetic Data Generation}

In lieu of high quality in vivo ground truth data we validate our technique first using synthetic data generated from measured optical characteristics of blood [7] and sub mucosa [30]. For simulating the interaction of light and tissue, the Monte Carlo for Multi-Layered media (MCML) simulation [31] has been an established method for simulating light transport through layered turbid media. To simulate tube like vessels we use the Mesh-based Monte Carlo (MMC) [32] because this develops a more expressive simulation framework that allows 3D mesh based structures, as well as refraction and reflection across boundaries of different refractive indices. We simulated MSI data using MMC which was subsequently filtered by applying known RGB camera spectral responses to create RGB images. The digital phantom, shown in Fig. 4, comprised a homogeneous block of soft tissue, bowel sub mucosa, with three superficial vessels containing either oxygenated or de-oxygenated blood. The three blood vessels had different uniform diameters of 2,1 and $0.5 \mathrm{~mm}$ and the top 


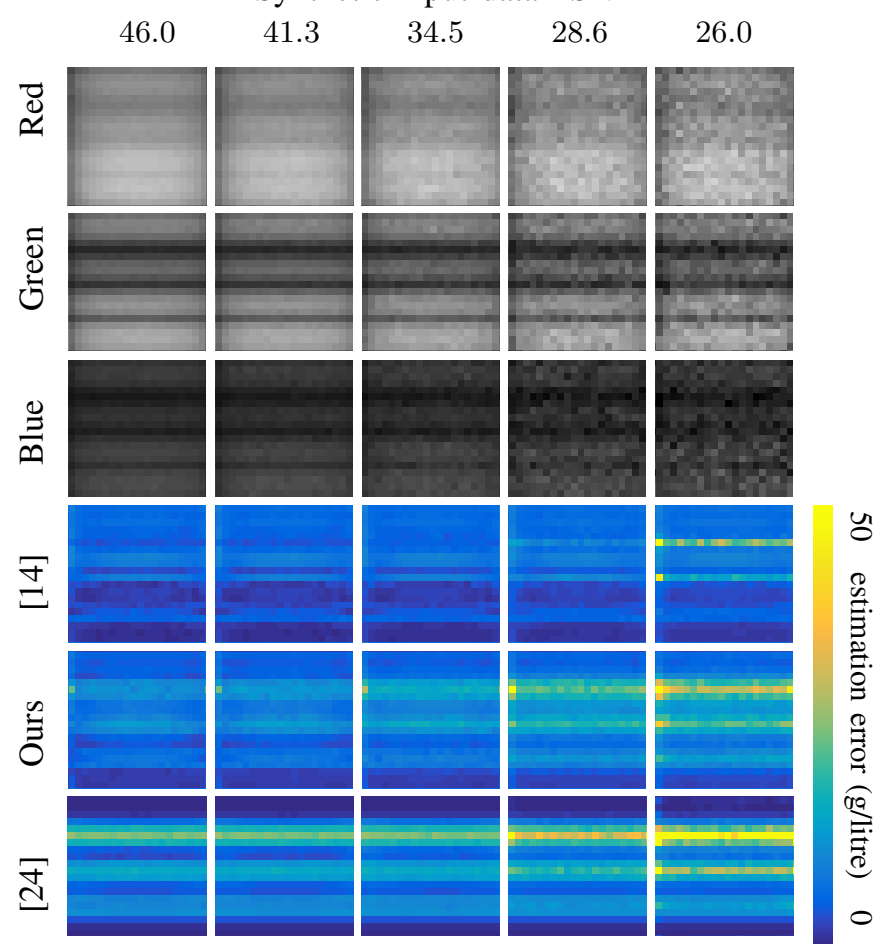

Fig. 5: Top three rows: example synthetic RGB responses for increasing levels of added noise, PSNR calculated with respect to zero added noise data. Lower three rows: visualisation of the mean absolute error in chromophore concentration for our method, Tikhonov regularised estimation [24] and a full multispectral approach [14].

edge of each vessel was at $0.1 \mathrm{~mm}$ below the surface of the tissue. This depth was chosen to balance the obfuscating effect of the surrounding soft-tissue volume while maintaining reasonable detectability of the vessels. While this may seem shallow, the high degree of scattering makes it increasingly difficult to detect vessels embedded more than $2 \mathrm{~mm}$ beneath the surface. However, this is typical for MSI which is known to be only capable of measuring a superficial layer of tissue. The optical characteristics of blood and colonic sub-mucosa were compiled from [7] and [30] respectively. The mesh was made denser near the blood vessel boundaries for smoother approximation of the curved vessel walls.

During MMC simulation, photons were generated at intervals of $10 \mathrm{~nm}$ across the visible range between $400 \mathrm{~nm}$ to $900 \mathrm{~nm}$. To detect the backscattered light, the final direction of travel for each photon was recorded leaving the bounds of the meshed region. Photons that did not exit through the side of the mesh that was illuminated were discarded as were photons leaving at angles to the surface too oblique to be detected by a detector placed at $10 \mathrm{~mm}$ above the illuminated surface. The simulated detector was placed close to the tissue surface because the light exiting the tissue is highly diffuse and placing the detector further from the tissue would have an exponential penalty in simulation time.

Image data for each wavelength band were then generated at the detector location. A total of $2 \times 10^{9}$ photons were generated uniformly distributed across the illumination surface with random initialisations. The number of photons was chosen by following MMC examples and documentation, as well as, from the practical constraint of the number of photons we could simulate in 5 weeks of simulation time. To simulate MSI data, we first filtered photons into spectral bands and then used the incident angle at the detector to further filter photons according to the camera model. Since, for computational reasons, the detector in our simulation environment had to be close to the tissue surface we simulated an orthographic camera. The orthographic camera model also avoids angular bias found in projective cameras, where light at the edge of the image exits the tissue at a much shallower angle than that at the centre. RGB images were generated by filtering the multispectral data using filters corresponding to sensitivity spectra of an RGB camera shown in Fig. 5.

\section{B. Synthetic Results}

We compare the results of our Bayesian estimation to a Tikhonov regularised estimation [24] as well as a full multispectral analysis [14]. The data used were the synthetic three vessel data, with vessels containing either $\mathrm{HbO}$ and $\mathrm{Hb}$. We added zero mean normally distributed noise to the input RGB and multispectral data at various levels to observe the performance deterioration in the presence of measurement or sensor noise. The results and examples from the RGB input are shown in Fig. 5 and also shown numerically in Fig. 6.

On the estimation of individual concentration values for $\mathrm{HbO}$ and $\mathrm{Hb}$, our method performs close to the hardware MSI method [14] and better than the Tikhonov method [24]. This constitutes a high degree of equivalent accuracy in estimating $\mathrm{THb}$. The $\mathrm{SO}_{2}$ estimation is particularly sensitive for Tikhonov estimation and the Bayesian method offers a notable improvement. While the mean improvement in $\mathrm{SO}_{2}$ estimation error is close to $10 \%$ at low noise levels there is a large reduction in the variance of this error. There is still a sizeable short fall in the accuracy compared to the hardware MSI. While not as capable as a multi-band approach our method at least seems to be generally indicative of tissue saturation and such relevant information may still be pertinent clinically.

The simulated imaging process to generate MS data using MC sampling is inherently noisy as it is the product of the passage of a finite number of photons, although the framework of [32] will eventually converge to the noise free result. Further it is difficult to eliminate the contribution of haemoglobin entirely when measuring the optical properties of tissue such as those for the sub-mucosa used in our model, this can result in a overly idealised ground truth haemoglobin distribution map, for which we assume that the submucosa is entirely vacant of $\mathrm{HbO}$ and $\mathrm{Hb}$. These are likely the dominant factors for why our reference method [14] does not achieve perfect results on the synthetic data.

\section{Results in the Presence of Calibration Noise}

The proposed framework is fundamentally dependent on the accuracy of the endoscopic sensor's spectral sensitivity calibration. In practice, a high quality calibration can be performed 


\begin{tabular}{cc|ccc|ccc}
\hline & Source & \multicolumn{2}{|c|}{ HbO Estimation Error (g/litre) } & \multicolumn{3}{c}{ Hb Estimation Error (g/litre) } \\
$\sigma$ & PSNR & {$[14]$} & Ours & {$[24]$} & {$[14]$} & Ours & {$[24]$} \\
\hline 0 & - & 0.23 & 0.44 & 0.82 & 0.94 & 1.23 & 3.71 \\
0.005 & 46.0 & $0.23 \pm 0.2$ & $0.51 \pm 0.3$ & $0.82 \pm 0.6$ & $0.94 \pm 0.6$ & $1.36 \pm 1.3$ & $3.79 \pm 3.1$ \\
0.006 & 44.2 & $0.24 \pm 0.2$ & $0.55 \pm 0.3$ & $0.83 \pm 0.6$ & $0.95 \pm 0.6$ & $1.42 \pm 1.4$ & $3.82 \pm 3.2$ \\
0.009 & 41.3 & $0.24 \pm 0.2$ & $0.64 \pm 0.4$ & $0.84 \pm 0.6$ & $0.95 \pm 0.6$ & $1.57 \pm 1.5$ & $3.83 \pm 3.2$ \\
0.013 & 37.9 & $0.25 \pm 0.2$ & $0.85 \pm 0.6$ & $0.90 \pm 0.7$ & $0.97 \pm 0.6$ & $1.95 \pm 1.9$ & $3.86 \pm 3.2$ \\
0.019 & 34.6 & $0.27 \pm 0.2$ & $1.20 \pm 1.0$ & $1.06 \pm 0.8$ & $1.04 \pm 0.7$ & $2.40 \pm 2.3$ & $3.88 \pm 3.3$ \\
0.027 & 31.4 & $0.31 \pm 0.3$ & $1.77 \pm 1.5$ & $1.72 \pm 0.5$ & $1.18 \pm 0.8$ & $3.04 \pm 3.0$ & $3.92 \pm 3.3$ \\
0.037 & 28.6 & $0.48 \pm 0.4$ & $2.71 \pm 2.4$ & $3.92 \pm 3.8$ & $2.16 \pm 1.7$ & $3.84 \pm 3.8$ & $4.61 \pm 4.1$ \\
0.050 & 26.0 & $1.83 \pm 1.7$ & $4.66 \pm 4.3$ & $11.3 \pm 11$ & $7.76 \pm 7.1$ & $6.66 \pm 6.6$ & $9.17 \pm 8.8$ \\
\hline
\end{tabular}

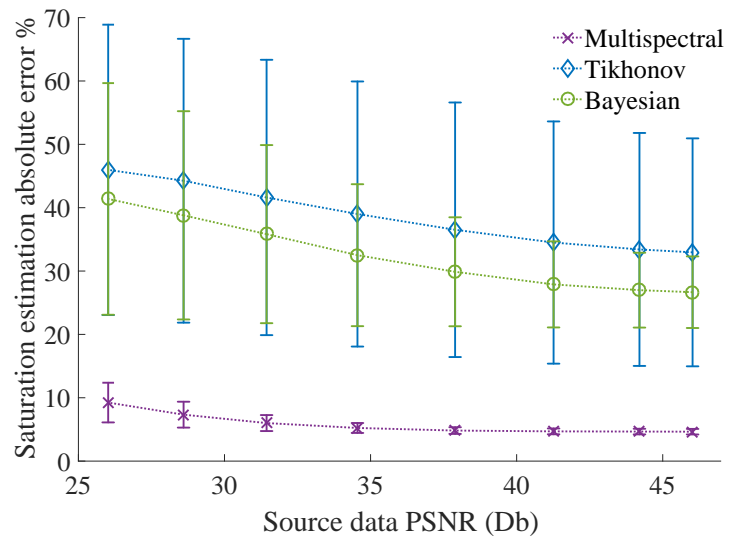

Fig. 6: Left: mean squared error (MSE) in estimating $\mathrm{HbO}$ and $\mathrm{Hb}$ concentrations ( $\mathrm{g} /$ /itre) under additive noise on synthetic data. NB numbers have been truncated for display but the variance is less than the mean error. Right: mean standard deviation in absolute error against the synthetic ground truth for increasing noise levels. Comparing our method (Bayesian) with [24] (Tikhonov) and [14] (Multispectral).

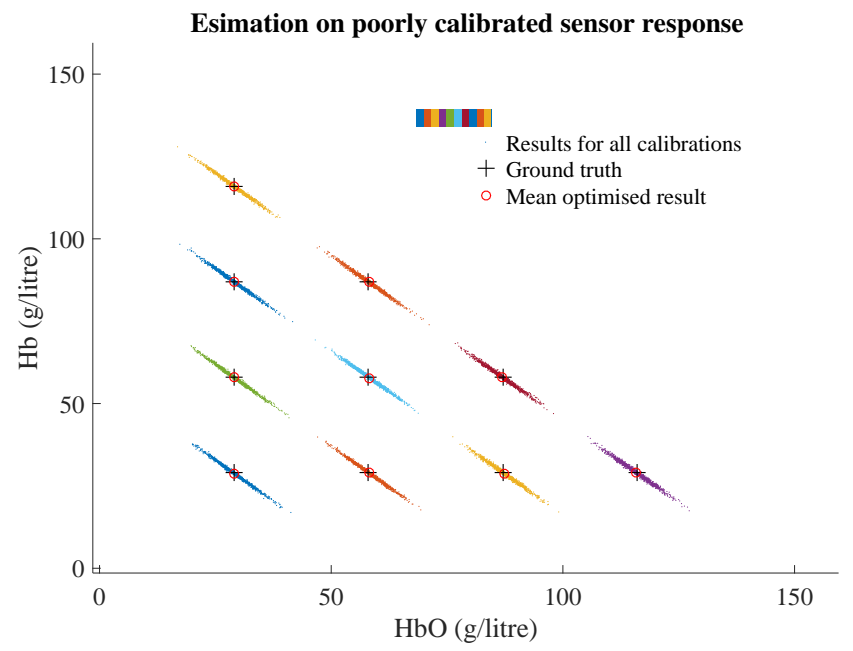

Fig. 7: The impact of noisy sensor calibration on Bayesian estimation is shown in this figure. The optimisation result using 1000 randomly sampled noisy sensor calibrations for 10 concentration pairings, here the noise level is $39.9 \mathrm{~dB}$ compared to ground truth.

using a monochromator and this would not need to be repeated often but at some points dependent on sensor characteristics and degradation over time [33]. Such a calibration can even be performed during manufacturing and remain accurate even for devices that are in regular clinical use [34], however, it is worth investigating the effect of a poor calibration for a more complete numerical understanding of our method's characteristics.

We chose to simulate incorrect calibrations for numerical investigation. Practically these could arise if calibration is performed by using low-cost solutions such as colour matching on Macbeth colour charts with known reflectance [35]. Images of such objects can be used to estimate the endoscopic sensor's response similarly to how geometric calibration is performed [35]. Notably, calibration with a monochromator can achieve much better accuracy when possible.
To assess the impact of poor calibration, we utilised reflectance spectra for 24 colour swatch chart [36] to simulate a RGB camera response under additive zero mean Gaussian noise. For each of the 24 swatches we generated a $256 \times 256$ patch measurement and then ran the calibration on the noisy data. We performed 10000 synthetic calibrations to numerically determine the distribution of calibration errors over wavelength, using this distribution we generated realistic miscalibrated sensor response curves. We generated multispectral data for 10 concentration pairings and then ran our method using 1000 randomly sampled mis-calibrated sensor response data.

We show the estimation for the 10 ground truth concentration pairings in Fig.7, for the calibration noise level of 39.9dB. This was chosen as it was the point at which errors noise began to be observable in the case of measurement noise. Visible in the results is the distribution of the error such that for any given ground truth value the errors are negatively correlated when comparing $\mathrm{HbO}$ and $\mathrm{HbO}$. This means that an incorrect calibration would produce results that are still fairly reliable for measuring the $\mathrm{THb}$ as this measure is based on the sum of the two values. However, the $\mathrm{SO}_{2}$ estimation is going to be very sensitive to poor calibration data as the ratio of $\mathrm{HbO}$ to $\mathrm{HbO}$ varies quite a lot. As such it would be important to use a high accuracy method to capture camera spectral calibration, such as that afforded by monochromator based methods.

\section{In vivo Data Acquisition}

To evaluate our method, we used data from a porcine study where multispectral imaging was performed on tissue undergoing periods of artificially induced restricted blood flow [37]. The original procedure was undertaken with the assistance of Northwick Park Institute for Medical Research. The surgical procedure was conducted under UK Home Office personal animal licence (PIL) No. 70/24843 and project licence (PPL) No. 8012639.

The multispectral imaging hardware has been described previously [37]. It comprised a $30^{\circ}$ (Karl STORZ); a LCTF (Varispec; CRi); 50mm focal length achromatic imaging lens 

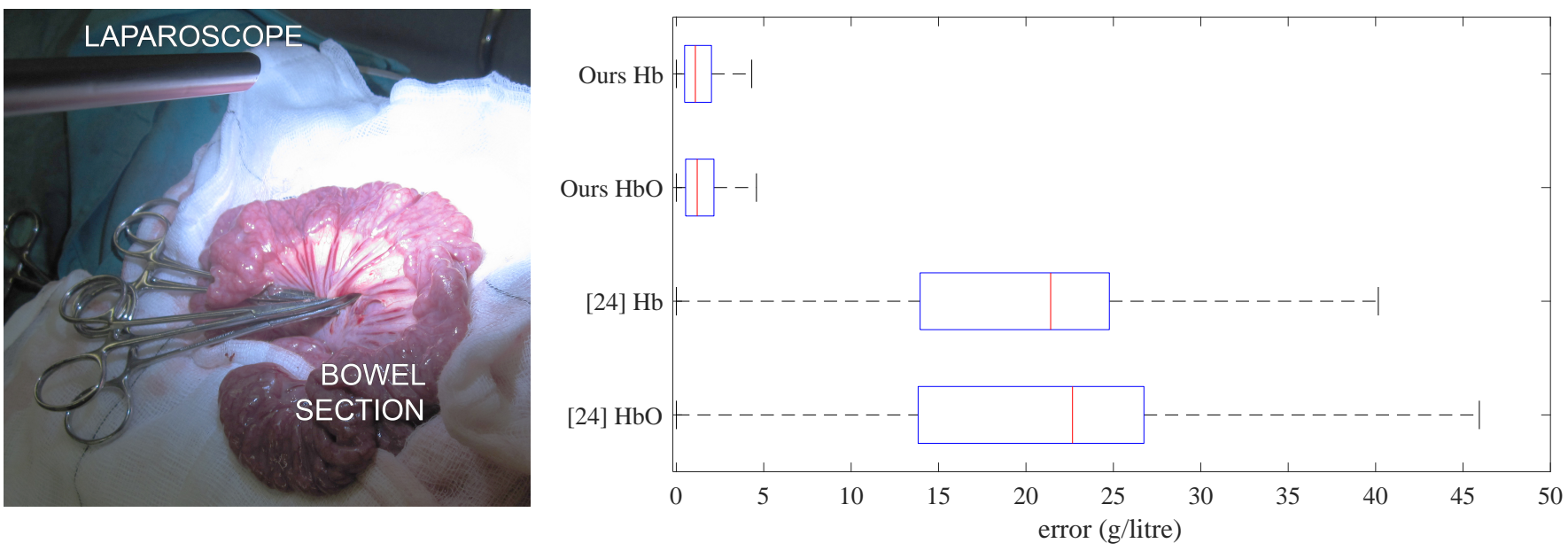

Fig. 8: Left: In vivo experimental configuration for imaging a section of porcine bowel. Right: Comparison of the absolute error for $\mathrm{HbO}$ to $\mathrm{HbO}$ concentration estimation, on in vivo data for our method and [24] estimation, against the multispectral ground truth.

(Thorlabs) and monochrome camera (DCU223M; Thorlabs Ltd., UK). Illumination was from a Xenon surgical light source (Xenon 300; Karl STORZ). The camera and laparoscope were held by adjustable arms whose pose could be locked in place to avoid camera shake. Multispectral data were captured serially in non-overlapping bands $10 \mathrm{~nm}$ wide from $500 \mathrm{~nm}$ to $620 \mathrm{~nm}$, the integration time at the sensor was $200 \mathrm{~ms}$ per band limited capture, requiring in a total of approximately 7 seconds for a full capture, after including filter switching and data readout times. To compensate for the tissue motion during from the long capture times for each complete multispectral capture, all band limited image were registered after acquisition [17].

The experiment imaged a section of porcine small bowel (45kg domestic white pig) under 4 different restricted blood flow configurations induced by occluding vessels for up to 15 minutes with rubber shod clamps. Blood flow was potentially restricted at two sites and 58 multispectral captures were made with the blood flow either fully restricted (both sites clamped) partially restricted (one site clamped) or unimpeded. Using the method of [14] we created a ground truth total haemoglobin estimation for each multispectral set, masking out regions where the coefficient of determination (CoD) of this fit was lower then 0.5. To create our input RGB data we composited the multispectral data into a three channel image using a known camera response curve. The imaging configuration can be seen in Fig. 9, a section of bowel is exposed and some vessels are clamped to restrict blood flow.

\section{E. In vivo Results}

We compare our Bayesian method described in this paper and the Tikhonov regularised estimation against the multispectral results and illustrate this in Fig. 8, 9 and 10. We observed a strong correspondence between the multispectral ground truth and both our method and Tikhonov regularised estimation, however, our method is more accurate in correctly estimating the ratio between the concentration of $\mathrm{HbO}$ to $\mathrm{Hb}$ as shown in Fig. 8. The bias of the [24] estimation is shown in the corresponding error histogram of Fig. 9 as twin peaks either side of zero, there are similar much smaller peaks visible in the error histogram for out method however most of the mass for our methods error is concentrated around zero. Numerically, we evaluate our method and [24] against the result from [14] as we aim to closely approximate this result. We measured the peak signal-to-noise ratio (PSNR) for our method as $45.9 \pm 8 \mathrm{~dB}$ for THb estimation, $28.6 \pm 3 \mathrm{~dB}$ for $\mathrm{SO}_{2}$ estimation and $35.8 \pm 4 \mathrm{~dB}$ for the overall raw $\mathrm{Hb}$ and $\mathrm{HbO}$ concentration values. In contrast, the Tikhonov regularised estimation yielded a PSNR of $38.2 \pm 2 \mathrm{~dB}$ for $\mathrm{THb}$ estimation, $8.2 \pm 1 \mathrm{~dB}$ for $\mathrm{SO}_{2}$ estimation and $16.8 \pm 1 \mathrm{~dB}$ for the overall estimation. As observed in the synthetic experiments, we see a marked improvement in the accuracy of saturation estimation for our method over the Tikhonov regularised approach. The improvement in the $\mathrm{THb}$ estimation is less significant when comparing against the multispectral result as ground truth. This contrast in performance is shown in Fig. 10. We measured the Pearson correlation coefficient ${ }^{2}$ of the haemoglobin concentrations from our Bayesian method and the Tikhonov approach [24] against the MSI data estimated using a hardware system [14]. For our method we measured a correlation coefficient of 0.996 and for [24] a correlation coefficient of 0.766 . For both, the confidence level on the correlation was above $99.9 \%$ confidence level and sample sizes were greater than $3 \times 10^{7}$ data points. The stronger correlation for our method explains why we observe a smaller error in saturation estimation in comparison to the Tikhonov regularised estimation.

A comparison of the estimation errors for $\mathrm{THb}$ and $\mathrm{SO}_{2}$ is shown in Fig. 9, illustrated here is how the $\mathrm{SO}_{2}$ estimation error seems to be lower in areas corresponding to vasculature,

\footnotetext{
${ }^{2}$ calculated using MATLAB corrcoef function
} 

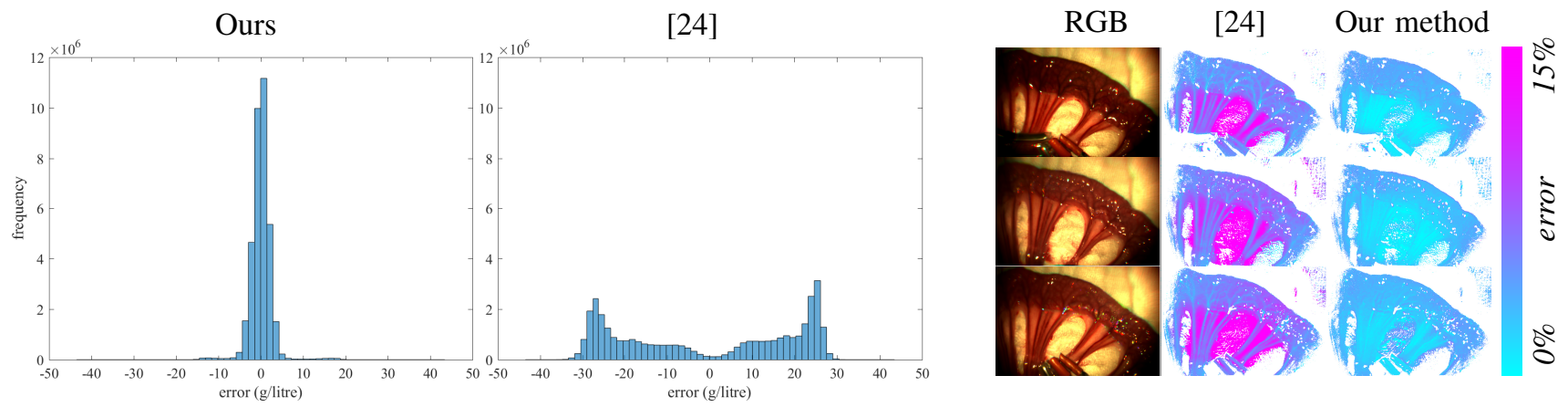

Fig. 9: Left: Error histograms for our method and [24] against [14] (used as ground truth) combined for HbO and Hb. Our method's error is more closely grouped around zero. Right example absolute error maps for the saturation estimation with RGB view of the surgical site for reference, visible is the lower error in areas corresponding to vasculature (higher perfusion).

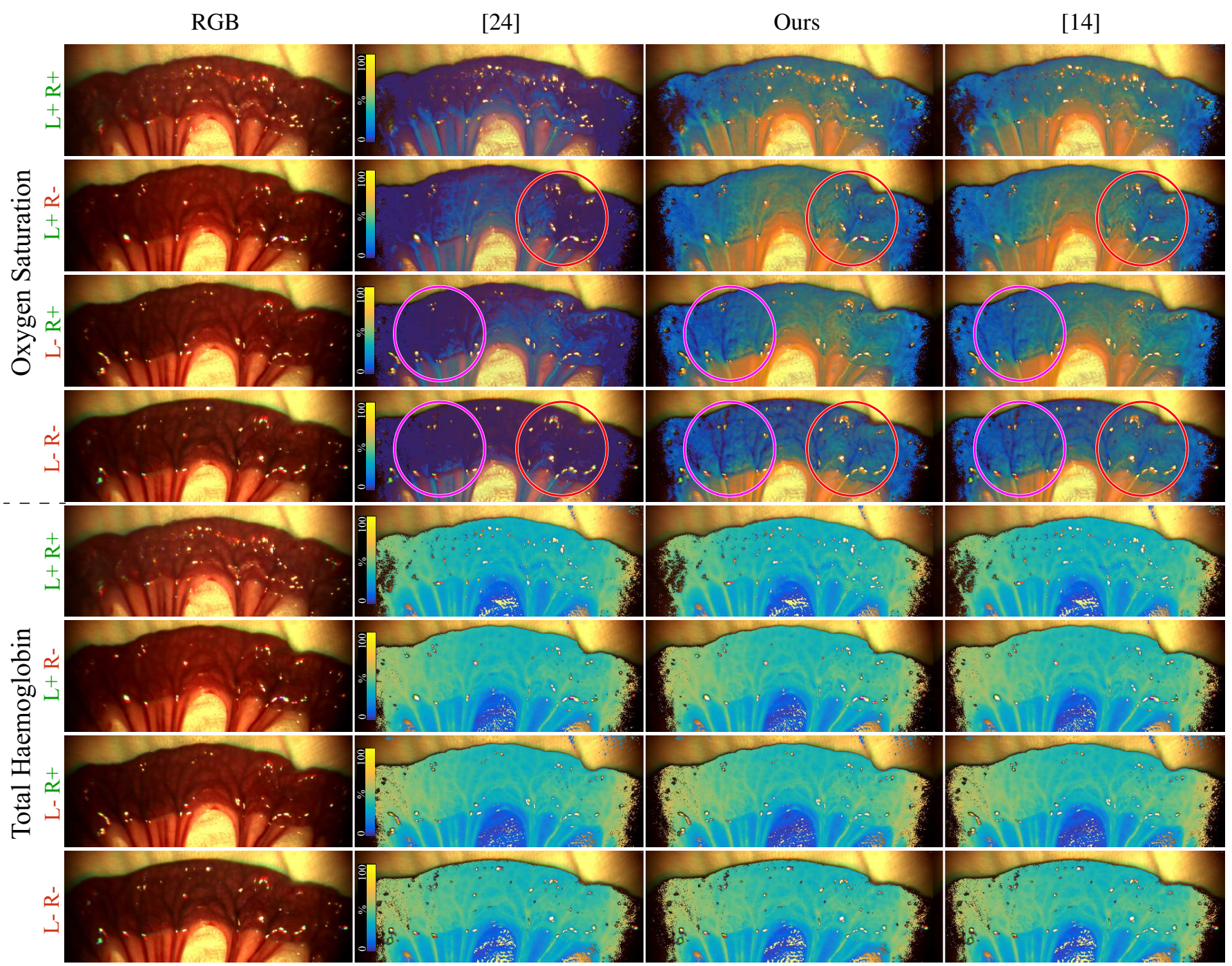

Fig. 10: $\mathrm{SO}_{2}$ and $\mathrm{THb}$ maps corresponding to the four configurations of the in vivo experiments, clamping vessels leading to either the left or the right of the bowel as viewed, green $(+)$ corresponds to clamp open and red $(-)$ corresponds to clamp closed. Our method (Bayes) is shown with the multispectral method of [14] and Tikhonov regularised estimation [24]. Maps of $\mathrm{SO}_{2}$ distribution are displayed at the top and $\mathrm{THb}$ maps are displayed to the bottom, both overlaid on a reference RGB view as visible in the left most column. For the $\mathrm{SO}_{2}$ maps they have been overlaid by alpha blending using the $\mathrm{THb}$ as an alpha value, regions are also highlighted corresponding to the part of the bowel with restricted circulation. 
and worse in regions where there is expected to be a lower $\mathrm{THb}$ value. This is to be expected because when there are low concentrations of both chromophores, slight errors in estimation of either can lead to a significant change in the ratio of one to the other. The THb estimation for our Bayesian method showed a small improvement compared to the Tikhonov regularised estimation. For both the raw concentration values ( $\mathrm{HbO}$ and $\mathrm{Hb})$ and the composite measures ( $\mathrm{THb}$ and $\left.\mathrm{SO}_{2}\right)$, our method produced a lower mean absolute error and the estimation error for our method also had a lower variance, when comparing against the Tikhonov approach.

It is possible to observe similar localisation patterns in the saturation of blood within tissue in our results as to that of hardware MSI analysis. In Fig. 10 highlighted are the parts of the small bowel that correspond to the tops of blood vessels that were either restricted or unimpeded, in the cases where blood flow is restricted there can be seen a noticeable drop in the oxygenation of these regions. While we have shown that the result of our method is numerically similar to the result from MSI, in Fig. 10 we can see how the visualisation of the result from our method is also visually similar to the visualisation of the MSI result.

\section{Discussion AND CONCLUSIONS}

We have presented a framework for MSI using only a limited number of wide-band measurements, which has the potential to integrate within current endoscopic systems with RGB sensors. Our algorithm is able to run at standard video frame rate $(24 \mathrm{fps})$ and provide information on haemoglobin concentrations within tissue in real-time. The results we have shown are commensurate with those obtained through hardware MSI and while our technique does not achieve the same accuracy on simulated data, its potential for providing additional information within existing endoscopic imaging systems could be clinically valuable. Additionally, while MSI using higher numbers of wavelength limited acquisitions, achieves better spectral discrimination, it suffers from misalignment in the data cube when imaging dynamic tissue and our method could be important in such applications.

One limitation of our method is that it requires the camera and illumination source to be at a fixed distance from the tissue surface because we use this assumption in order to simplify the estimation term. We need to perform additional investigations into the effect of this assumption. Speculatively, changing the imaging distance would result in over and underestimation of the concentration of chromophores within tissue. This would arise from the illumination source not being equivalently diffuse as the light leaving tissue. This could be compensated in theory by modelling the relationship to depth and then using computer vision for 3D reconstruction to inform our estimation framework. As well as depth, surface normal information, which could be obtained with dense $3 \mathrm{D}$ reconstruction methods, may allow us to incorporate directional bias of light leaving the tissue surface to further improve our technique. An important note about our Bayesian framework is that it can be extended to incorporate additional constraints. As well as depth, other potential regularisation terms about local spatial or temporal structure could be introduced. The current implementation operates on a per-pixel basis but it is clear that in endoscopic images neighbouring points on the tissue surface are related and represent physiological structures like blood vessels or tissues from different organs. Combining information, for example from image segmentation, within the framework could allow better regularisation and estimation with multiple pixel measurements rather than the current single measurement approach.

While our experiments have shown that the proposed method is promising and can provide information comparable to hardware MSI, it is important to note that additional experimentation is necessary to understand both the quantities we are trying to estimate and the clinical applications that may benefit. We plan to carry out these studies both in controlled experimental conditions and within the clinical setting to further understand how our method may be clinically useful during treatment.

\section{ACKNOWLEDGEMENTS}

We thank Nvidia for donating a Quadro 6000 which was used for this work. We thank all of the staff at Northwick Park Institute for Medical Research and Shobhit Arya, Mohan Singh and George Hanna. Neil Clancy is supported by an Imperial College Junior Research Fellowship. The work was also supported by ERC grant 242991. Danail Stoyanov receives funding from the EPSRC (EP/N013220/1, EP/N022750/1), the EU-FP7 project CASCADE (FP7-ICT-2913-601021) and the EU-Horizon2020 project EndoVESPA (H2020-ICT-2015688592).

\section{REFERENCES}

[1] D. Stoyanov, "Surgical vision," Annals of Biomedical Engineering, vol. 40, no. 2, pp. 332-345, 2011.

[2] A. Darzi and S. Mackay, "Recent advances in minimal access surgery," British Medical Journal, vol. 324, no. 7328, p. 31, 2002.

[3] D. J. Mirota, M. Ishii, and G. D. Hager, "Vision-based navigation in image-guided interventions," Annual Review of Biomedical Engineering, vol. 13, no. 1, pp. 297-319, 2011.

[4] A. Taruttis, A. Rosenthal, M. Kacprowicz, N. C. Burton, and V. Ntziachristos, "Multiscale multispectral optoacoustic tomography by a stationary wavelet transform prior to unmixing," IEEE Transactions on Medical Imaging, vol. 33, no. 5, pp. 1194-1202, 2014.

[5] L. V. Wang and S. Hu, "Photoacoustic tomography: In vivo imaging from organelles to organs," Science, vol. 335, no. 6075, pp. 1458-1462, 2012.

[6] J.-L. Boulnois, "Photophysical processes in recent medical laser developments: A review," Lasers in Medical Science, vol. 1, no. 1, pp. 47-66, 1986.

[7] N. Bosschaart, G. J. Edelman, M. C. G. Aalders, T. G. van Leeuwen, and D. J. Faber, "A literature review and novel theoretical approach on the optical properties of whole blood," Lasers Med Sci, vol. 29, no. 2, pp. 453-479, 2013.

[8] B. S. Sorg, B. J. Moeller, O. Donovan, Y. Cao, and M. W. Dewhirst, "Hyperspectral imaging of hemoglobin saturation in tumor microvasculature and tumor hypoxia development," Journal of biomedical optics, vol. 10, no. 4, pp. 044 004-044004, 2005.

[9] N. T. Clancy, S. Saso, Danail, V. Sauvage, D. J. Corless, M. Boyd, D. E. Noakes, M.-Y. Thum, S. Ghaem-Maghami, J. R. Smith, and D. S. Elson, "Multispectral imaging of organ viability during uterine transplantation surgery," in Advanced Biomedical and Clinical Diagnostic Systems XII. SPIE-Intl Soc Opt Eng, 2014.

[10] S. P. Nighswander-Rempel, R. A. Shaw, J. R. Mansfield, M. Hewko, V. V. Kupriyanov, and H. H. Mantsch, "Regional variations in myocardial tissue oxygenation mapped by near-infrared spectroscopic imaging," Journal of Molecular and Cellular Cardiology, vol. 34, no. 9, pp. 1195 - 1203, 2002. 
[11] R. Sznitman, S. Billings, D. Rother, D. Mirota, Y. Yang, J. Handa, P. Gehlbach, J. U. Kang, G. D. Hager, and R. Taylor, "Active multispectral illumination and image fusion for retinal microsurgery," in Information Processing in Computer-Assisted Interventions. Springer Science Business Media, 2010, pp. 12-22.

[12] E. Claridge, D. Hidović-Rowe, P. Taniere, and T. Ismail, "Quantifying mucosal blood volume fraction from multispectral images of the colon," in Medical Imaging. International Society for Optics and Photonics, 2007, pp. 65 110C-65 110C.

[13] M. B. Bouchard, B. R. Chen, S. A. Burgess, and E. Hillman, "Ultrafast multispectral optical imaging of cortical oxygenation, blood flow, and intracellular calcium dynamics," Optics express, vol. 17, no. 18, pp. $15670-15678,2009$

[14] N. T. Clancy, D. Stoyanov, D. R. C. James, A. D. Marco, V. Sauvage, J. Clark, G. Z. Yang, and D. S. Elson, "Multispectral image alignment using a three channel endoscope in vivo during minimally invasive surgery," Biomed. Opt. Express, vol. 3, no. 10, pp. 2567-2578, 2012.

[15] S. J. Wirkert, N. T. Clancy, D. Stoyanov, S. Arya, G. B. Hanna, H.P. Schlemmer, P. Sauer, D. S. Elson, and L. Maier-Hein, Endoscopic Sheffield Index for Unsupervised In Vivo Spectral Band Selection. Springer International Publishing, 2014, pp. 110-120.

[16] N. T. Clancy, D. Stoyanov, V. Sauvage, D. James, G.-Z. Yang, and D. S. Elson, "A triple endoscope system for alignment of multispectral images of moving tissue," in Biomedical Optics and 3-D Imaging. Optical Society of America, 2010, p. BTuD27.

[17] D. Stoyanov, A. Rayshubskiy, and E. Hillman, "Robust registration of multispectral images of the cortical surface in neurosurgery," in Biomedical Imaging (ISBI), 2012 9th IEEE International Symposium on. IEEE, 2012, pp. 1643-1646.

[18] G. Jones, N. Clancy, S. Arridge, D. Elson, and D. Stoyanov, Deblurring Multispectral Laparoscopic Images. Cham: Springer International Publishing, 2014, pp. 216-225.

[19] X. Du, N. Clancy, S. Arya, G. B. Hanna, J. Kelly, D. S. Elson, an D. Stoyanov, "Robust surface tracking combining features, intensity and illumination compensation," International Journal of Computer Assisted Radiology and Surgery, vol. 10, no. 12, pp. 1915-1926, 2015.

[20] I. Meglinski and S. Matcher, "Computer simulation of the skin reflectance spectra," Computer Methods and Programs in Biomedicine, vol. 70, no. 2, pp. 179-186, 2003.

[21] G. I. Petrov, A. Doronin, H. T. Whelan, I. Meglinski, and V. V. Yakovlev, "Human tissue color as viewed in high dynamic range optical spectral transmission measurements," Biomedical Optics Express, vol. 3, no. 9, p. 2154, 2012.

[22] I. Nishidate, T. Maeda, K. Niizeki, and Y. Aizu, "Estimation of melanin and hemoglobin using spectral reflectance images reconstructed from a digital RGB image by the wiener estimation method," Sensors, vol. 13, no. 6, pp. 7902-7915, 2013

[23] Y. Fawzy, S. Lam, and H. Zeng, "Rapid multispectral endoscopic imaging system for near real-time mapping of the mucosa blood supply in the lung," Biomedical Optics Express, vol. 6, no. 8, p. 2980, 2015.

[24] G. Jones, N. T. Clancy, Y. Helo, S. Arridge, D. S. Elson, and D. Stoyanov, Inference of Tissue Haemoglobin Concentration from Stereo RGB. Springer International Publishing, 2016, pp. 50-58

[25] D. T. Delpy, M. Cope, P. van der Zee, S. Arridge, S. Wray, and J. Wyatt, "Estimation of optical pathlength through tissue from direct time of flight measurement," Physics in Medicine and Biology, vol. 33, no. 12, pp. 1433-1442, 1988.

[26] C. E. Thorn, S. J. Matcher, I. V. Meglinski, and A. C. Shore, "Is mean blood saturation a useful marker of tissue oxygenation?" AJP: Heart and Circulatory Physiology, vol. 296, no. 5, pp. H1289-H1295, 2009.

[27] A. K. Dunn, A. Devor, A. M. Dale, and D. A. Boas, "Spatial extent of oxygen metabolism and hemodynamic changes during functional activation of the rat somatosensory cortex," Neurolmage, vol. 27, no. 2, pp. 279-290, 2005.

[28] V. Morozov, "Regularization of incorrectly posed problems and the choice of regularization parameter," USSR Computational Mathematics and Mathematical Physics, vol. 6, no. 1, pp. 242-251, 1966.

[29] R. Bro and S. D. Jong, "A fast non-negativity-constrained least squares algorithm," J. Chemometrics, vol. 11, no. 5, pp. 393-401, 1997.

[30] A. N. Bashkatov, E. A. Genina, V. I. Kochubey, V. S. Rubtsov, E. A Kolesnikova, and V. V. Tuchin, "Optical properties of human colon tissues in the $350-2500 \mathrm{~nm}$ spectral range," Quantum Electronics, vol. 44, no. 8, pp. 779-784, 2014.

[31] L. Wang, S. L. Jacques, and L. Zheng, "MCML-monte carlo modeling of light transport in multi-layered tissues," Computer Methods and Programs in Biomedicine, vol. 47, no. 2, pp. 131-146, 1995.
[32] Q. Fang, "Mesh-based monte carlo method using fast ray-tracing in plücker coordinates," Biomed. Opt. Express, vol. 1, no. 1, p. 165, 2010.

[33] "EMVA standard 1288: Standard for Characterization of Image Sensors and Cameras," European Machine Vision Association, no. 3.0, 2010.

[34] M. Hohmann, R. Kanawade, F. Klämpfl, A. Douplik, J. Mudter, M. F. Neurath, and H. Albrecht, "In-vivo multispectral video endoscopy towards in-vivo hyperspectral video endoscopy," Journal of Biophotonics, p. preprint, 2016.

[35] J. Y. Hardeberg, H. Brettel, and F. J. M. Schmitt, "Spectral characterization of electronic cameras," in Electronic Imaging: Processing, Printing, and Publishing in Color, J. Bares, Ed. SPIE-Intl Soc Optical Eng, 1998

[36] D. Pascale. (2012, Accessed: May 2016) Color Checker Data. Online: http://www.babelcolor.com/colorchecker-2.htm\#CCP2_data.

[37] N. T. Clancy, S. Arya, D. Stoyanov, M. Singh, G. B. Hanna, and D. S. Elson, "Intraoperative measurement of bowel oxygen saturation using a multispectral imaging laparoscope," Biomedical Optics Express, vol. 6, no. 10 , p. 4179,2015 . 